Methods: Study group included 88 patients with RA, their age was $53 \pm 11,5$, $79.3 \%$ of women, $89.8 \%$ of RF "+," DAS28 $5.2 \pm 1.2$, receiving DMARDs (methotrexate $59.5 \%$ and leflunomide $19.8 \%$ ), who were administered with tofacitinib $5 \mathrm{mg} 2$ times a day due to inefficacy or intolerance of biological DMARDs. There were assessed the pain severity using Brief pain inventory (BPI) questionnaire, the presence of neuropathic pain component (NPC) using PainDETECT questionnaire and signs of CS using Central Sensitisation Inventory (CSI) questionnaire at early time after tofacitinib administration, RA activity using DAS28 after 3 and 6 months. Results: The mean pain severity at baseline was $5.3 \pm 2.0$ according to the visual analogue scale (VAS $0-10$ ), $51.1 \%$ of patients had signs of central sensitization (CSI $\geq 40$ ), $15.9 \%$ had NPC (PainDETECT $\geq 18$ ). 7 days after tofacitinib intake there was statistically reliable reduction of pain severity - up to $4.1 \pm 1.8$ $(p<0.05)$ and CS $-C S I$ from $40.4 \pm 13.5$ to $36.5 \pm 12.5(p=0.01)$. After 28 days, the effect was higher: the pain level (VAS) was $2.8 \pm 1.6(p=0.000)$, PainDETECT decreased from $11.8 \pm 5.6$ to $6.8 \pm 3.1(p=0.000), C S I-$ to $31.6 \pm 13.9(p=0.000)$. DAS28 after 3 and 6 months was $3.7 \pm 1.3$ and $3.6 \pm 1.2$. The number of patients with pain decrease of $\geq 50 \%$ after 28 days of therapy was $59.9 \%$. Low RA activity after 3 months. (DAS28 $\leq 3.2$ ) was achieved in $64.4 \%$ of patients. There was a clear correlation between the number of patients with significant pain reduction at 28 days and the number of patients with low RA activity after 3 and 6 months $\left(r_{s}=0.548, p=0.000 ; r_{s}=0.790, p=0.000\right)$. Six patients withdrew from the study due to inefficacy or social reasons. There were no serious adverse reactions.

Conclusion: The application of JAK inhibitor tofacitinib allows to reach a fast analgesic effect and reduce CS signs. An early clinical response to tofacitinib (pain relief) predicts a decrease in RA activity after 3 and 6 months of the therapy. Limitation: Open-label observatory study.

Disclosure of Interests: None declared

DOI: 10.1136/annrheumdis-2021-eular.2902

\section{AB0136 ASSESSING THE RELATIONSHIP AMONG OBESITY, GENETIC POLYMORPHISM, AND CLINICAL PARAMETERS IN PATIENTS WITH RHEUMATOID ARTHRITIS}

K. Yoshida ${ }^{1}$, S. Hatachi ${ }^{1}$, S. Takahashi ${ }^{1}$, N. Amano ${ }^{1}$, M. Katayama ${ }^{1}$, M. Sibata ${ }^{2}$, T. Saito ${ }^{2}$, S. Kumagai ${ }^{1,2}$. ${ }^{1}$ Shinko Hospital, Center for Rheumatic Diseases, Kobe, Japan; ${ }^{2}$ Shinko Institute for Medical Research, Kobe, Japan

Background: Several study suggested body mass index(BMI) may influence development of rheumatoid arthritis(RA). There are conflicting reports concerning the impact of high BMI on development of RA, but several reports of obese on drug resistance and functional impairment. The relationship of genetic polymorphism on obesity is unclear in RA.

Objectives: To examine the relationship among BMI, genetic polymorphism of obesity, disease activity of RA, laboratory parameters, and therapeutic agent of RA.

Methods: We have carried out a retrospective observational study by systematically analyzing medical records of total 289 patients diagnosed with RA in Shinko Hospital between March 2016 and December 2019. We also conducted genotyping single nucleotide polymorphisms (SNPs) including FTO (rs1558902 and rs9939609), UCP1 (rs1800592), ADR2(rs1042713) and ADR3(rs4994) after informed consent. Obesity was defined as BMI over than 25 and patients were divided between obese ("Ob") and non-obese ("non-Ob"). These SNPs, DAS28CRP, laboratory parameters, methotrexate dose, use of biological DMARDs were compared between $\mathrm{Ob}$ and non-Ob patients.

Results: Of these 289 patients, $82.7 \%$ was female, mean age was 61.9 years and BMI was 22.4. Univariate logistic regression showed differences $(p<0.1)$ between $\mathrm{Ob}$ and non $\mathrm{Ob}$ groups in UCP1 gene mutation $63.6 \%$ vs $78 \%$, $\mathrm{P}=0.018)$, DAS(2.24 vs $1.99, \mathrm{P}=0.033)$,triglyceride abnormality(23.8\% vs $9.3 \%$, $\mathrm{P}=0.021), \mathrm{HDL}(56$ vs $71, \mathrm{P}=0.00009), \mathrm{HbA} 1 \mathrm{c}$ abnormality $(26.5 \%$ vs $12.1 \%$, $\mathrm{P}=0.019), \gamma \mathrm{GTP}(32$ vs $21, \mathrm{P}=0.00037), \mathrm{ALP}$ (253 vs 230, $\mathrm{P}=0.0058), \operatorname{ALT}(26.5$ vs $20, P=0.029$ ), and MTX dose( 6 vs $8, P=0.066)$. Multivariate logistic regression showed that $\mathrm{Ob}$ group was significantly associated with $\mathrm{HDL}(\mathrm{OR}=0.976,95 \% \mathrm{Cl}$ 0.958 to 0.995$)$, UCP1 gene mutation(OR=0.446, $95 \% \mathrm{Cl} 0.202$ to 0.984$), \gamma \mathrm{GT}-$ $\mathrm{P}(\mathrm{OR}=2.321,95 \% \mathrm{Cl} 1.269$ to 4.245$)$, and $\mathrm{MTX}$ dose(OR=0.866, 0.784 to 0.957$)$. Conclusion: Obesity in patients with RA had significant positive correlation with $\gamma$ GTP, and negative correlation with HDL, UCP gene mutation and MTX dose. Disclosure of Interests: None declared

DOI: 10.1136/annrheumdis-2021-eular.2962

\begin{tabular}{|l|l|}
\hline AB0137 & THE ASSOCIATION BETWEEN AUTOANTIBODY \\
& LEVELS AND THE OUTCOMES OF ANTI-TUMOUR \\
& NECROSIS FACTOR ALPHA TREATMENT IN \\
& RHEUMATOID ARTHRITIS - A RETROSPECTIVE \\
COHORT STUDY WITH TWO YEARS FOLLOW-UP
\end{tabular}

D. Santos Oliveira ${ }^{1,2}$, A. Martins ${ }^{1}$, F. R. Martins ${ }^{3}$, M. Rato $^{1}$, F. Oliveira Pinheiro ${ }^{1}$, D. Fonseca ${ }^{4}$, S. Garcia ${ }^{1}$, B. M. Fernandes ${ }^{1}$, C. Vaz ${ }^{1,2,5}$, M. Bernardes ${ }^{1,5}$,

L. Costa ${ }^{1} .{ }^{1}$ Centro Hospitalar Universitário de São João, Rheumatology
Department, Porto, Portugal; ${ }^{2}$ Faculty of Medicine, University of Porto, Center for Health Technology and Services Research (CINTESIS), Porto, Portugal; ${ }^{3}$ Centro Hospitalar Universitário do Algarve, Rheumatology Department, Faro, Portugal; ${ }^{4}$ Centro Hospitalar Vila Nova de Gaia / Espinho, Rheumatology Department, Vila Nova de Gaia, Portugal; ${ }^{5}$ Faculty of Medicine, University of Porto, Department of Medicine, Porto, Portugal

Background: In rheumatoid arthritis (RA), autoantibodies namely anticitrullinated protein antibodies (Anti-CCP) have prognostic value, independently predicting radiologic progression. However, the evidence is still controversial about how the autoantibody levels change over time and their role in treatments outcomes and in monitoring disease activity in RA.

Objectives: This study aimed to characterize the changes of autoantibodies levels (rheumatoid factor (RF) and Anti-CCP) over time and to explore the association between these autoantibodies and the outcomes of the first anti-tumour necrosis factor alfa (anti-TNF- $\alpha$ ) therapy as first biologic agent in RA.

Methods: An observational retrospective cohort study was conducted with two years of follow-up. Patients with diagnosis of RA according to American College of Rheumatology (ACR) criteria and registered on Rheumatic Diseases Portuguese Register (Reuma.pt) who started their first anti-TNF $\alpha$ agent (as first biologic) between 2003 and 2018 were included. Patients with positive RA (>30 Ul/mL) and/ or positive Anti-CCP (>10 U/mL) at their first visit were included. Demographic, clinical and laboratory data were obtained by consulting Reuma.pt. Disease Activity Score for 28 joints [DAS28(3v); DAS28(4v); DAS28(3v; C-Reactive Protein (CRP)) DAS28(4v; CRP), delta DAS28(4v)], Health Assessment Questionnaire (HAQ), delta $H A Q$, Anti-CCP and RF levels were assessed at baseline, 12 and 24 months Continuous variables are presented with mean, standard deviation, median, quartile 1 and quartile 3. Categorical variables are presented with absolute and relative frequencies. To examine the differences between Anti-CCP and RF levels at baseline, 12 months and 24 months the Wilcoxon test for paired samples was performed. In order to correlate the Anti-CCP and RF levels with DAS28 variables, delta DAS28(4v), HAQ and delta HAQ at baseline, 12 months and 24 months, a correlation coefficient, Spearman's coefficient, was used.

Results: A total of 116 patients (mean age of $50.2 \pm 10.4$ years old; $85.3 \%$ female) with RA were included with a median disease duration of 10.5 [5-18.5] years and a follow-up time of 8 [5-14] years. About $49 \%$ of patients were FR and Anti-CCP positivity, 38\% only FR positivity and 13\% only Anti-CCP positivity. At baseline, 64 $(55.2 \%)$ patients had an erosive disease and 50 (43.1\%) had extra-articular manifestations. Compared to the baseline (160[74.8-496]), FR levels decreased significantly at 12 months (121[49.1-321.8]) and 24 months (107.5[43.3-332]) with a $p=0.017$ and $p=0.029$, respectively. There were no differences in Anti-CCP levels over time. No correlation was found between FR/Anti-CCP levels and different DAS28 variables, DAS28(4v) delta, HAQ, and HAQ delta at 12 months and 24 months.

Conclusion: We found that in patients with RA treated with a first anti-TNF- $\alpha$ agent as first biologic, FR levels decreased at 12 months and 24 months follow-up. However, our study failed to demonstrate a correlation between autoantibodies levels and disease activity (DAS28 variables and delta DAS28(4v)), HAQ and delta $\mathrm{HAQ}$. In fact, previous research demonstrated that there is an association between autoantibodies levels and disease activity in RA, nonetheless not being static and increasing with signs of inflammation at baseline. So, further research with large samples is needed to explore this correlation considering the adjustment for confounding inflammatory variables, such as number of swollen or tender joints and morning stiffness.

Disclosure of Interests: None declared

DOI: 10.1136/annrheumdis-2021-eular.3218

\section{AB0138 A MOLECULAR SIGNATURE RESPONSE CLASSIFIER STRATIFIES SEROPOSITIVE RHEUMATOID ARTHRITIS PATIENTS BASED ON THEIR LIKELIHOOD OF INADEQUATE RESPONSE TO TNF INHIBITOR THERAPIES}

S. Cohen ${ }^{1}$, V. Strand ${ }^{2}$, E. Connolly-Strong ${ }^{3}$, J. Withers ${ }^{3}$, L. Zhang ${ }^{3}$, T. Mellors ${ }^{3}$, V. Akmaev ${ }^{3} .{ }^{1}$ Metroplex Clinical Research Center, Rheumatology, Dallas, United States of America; ${ }^{2}$ Stanford University, Division of Immunology/Rheumatology, Palo Alto, United States of America; ${ }^{3}$ Scipher Medicine Corporation, Science and Technology, Waltham, United States of America

Background: There is an urgent need for precision medicine in targeted therapy selection for the treatment of rheumatoid arthritis (RA). TNF inhibitor (TNFi) therapies are the most prescribed targeted therapy for RA patients, yet the majority of patients fail to achieve a clinically meaningful response using this medication class. A blood-based molecular signature test evaluates RNA and clinical metrics to stratify RA patients based on their likelihood of having an inadequate response to TNFi therapies. ${ }^{1}$ Patients unlikely to respond to TNFi therapies can be directed to a different treatment option such as a JAK inhibitor, thus reducing the time needed to identify an effective therapy, improving confidence in and adherence to treatment, and increasing the patients' chance of reaching treat-to-target goals. 